\title{
Detection and Segmentation of Human Face
}

\author{
Nidhal K. El Abbadi', Ali Abdul Azeez Qazzaz ${ }^{2}$ \\ Computer Science Department, Education College, University of Kufa, Najaf, Iraq ${ }^{1,2}$
}

\begin{abstract}
Detection and segmentation of faces from an image is a crucial problem that has gained importance, face detection and segmentation play the main role in the face recognition systems. There are many difficulties should be solved to make the face detection and segmentation algorithm successful. The face skin has special colors range as well as special textures that can be detected by using texture recognition algorithms which recognized skin from the background. In this paper we introduced a new method for face detection and segmentation based on face color, we uses the $\mathrm{YCbCr}$ color space as a method to segment image to many regions. Gray level co-occurrence matrix used to extract the important features represent the skin, and then Tamura texture used to remove all the non skin blobs which is recognized as skin by GLCM. The proposed algorithm tested with many images and it was successfully recognize the images with faces from images without faces. The proposed algorithm has high efficient in detected faces and segmented faces from the background. The accuracy of this algorithm more than $99 \%$ in detection faces and also segment its. The proposed algorithm forms a prerequisite for any practical verification system using face as the main attribute.
\end{abstract}

Keywords: segmentation, face detection, GLCM, skin color, Tamura.

\section{INTRODUCTION}

The face of a human being conveys a lot of information about identity and emotional state of the person. Face detection in a complex scene and its segmentation from the background forms a prerequisite for any practical verification system using face as the main attribute. So, robust detection and segmentation forms the first step in building a fool proof system.

In the few decades the human face detection and segmentation emerge as an active research. There are many applications for this field such as face identification system, face tracking, video surveillance and security control system, and human computer interface. The preprocessing for all these applications is the face segmentation. There are many factors faces the process of face detection and segmentation such as the variation of face expression, skin color and may be the present and absent of some objects such as the mustache and glasses in addition to the variation of illuminate, face direction, color background, and distance between camera and subject [9]. Face detection is the process of finding faces in image and when detection the faces then it should be determine the face location and its content. Face detection is the interest step in the process of automatic analysis and face recognizing.

Image segmentation is the crucial problem in image analysis and image understanding. The face segmentation is an essential step of face recognition system since most face classification techniques tend to only work with face images. Therefore face segmentation has to correctly extract only face part of given large image [1].

The corner stone of face detection and segmentation is the human skin color which is used as effectively feature for face detection. Although skin color differs among individuals, several studies have shown that the major difference exists in the intensity rather than the chrominance. Several color spaces have been used to label skin pixels including RGB, NRGB (normalized RGB), HSV, YCrCb, CIE-XYZ, CIE- LUV.
Face detection approaches can be classified into features based approaches and image based approaches.

there are many researchers' works in this field, some of them are:

H. C. Vijay Lakshmi and S. PatilKulakarni, presented an Algorithm based on color space, they combine HSI and YCbCr along with Canny and Prewitt edge detection techniques. This technique improve an approaches suggested by other researchers [4]

K. Satheesh, I. Laurence Aroquiaraj, proposed algorithm called Hit and Missed Transformed (HMT) for face segmentation based on Mathematical Morphological Operator. The HMT determined the features for gray image to use it as a base of face segment [7].

El Abbadi, N.K., and A.H. Miry, Suggested new method to segment the skin lesions based on histogram thresholding. The input image filtered to remove most of segmentation problems such as variety in color, shapes, size, and other. Mathematical morphology used to enhance the image [2]

\section{METHODS AND MATERIALS}

In this proposal, the input image is colored image which transformed to ( $\mathrm{YCbCr}$ ) space color image by using the following relation:

$$
\left[\begin{array}{l}
y \\
C_{b} \\
C_{r}
\end{array}\right]=\left[\begin{array}{c}
16 \\
128 \\
128
\end{array}\right]+\left[\begin{array}{ccc}
0.299 & 0.587 & 0.114 \\
-0.168 & -0.331 & 0.5 \\
0.5 & -0.418 & -0.081
\end{array}\right] \times\left[\begin{array}{c}
\text { red } \\
\text { green } \\
\text { blue }
\end{array}\right]
$$

The important factor in this process is the $(\mathrm{Cr})$ component, which plays the main role in the face segmentation process; Fig. 1 shows the transformed process of image 


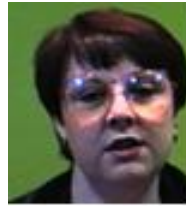

A

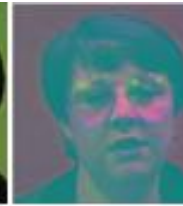

B

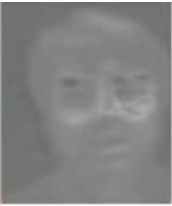

C
Fig. 1: transformed image. A. color image. B. YCbCr image. C. $\mathrm{Cr}$ image

Binary image is created from the $\mathrm{Cr}$ component; this is done by comparing each pixel value in the image with threshold (Cr mean). This step regards as the skin detector according to the mean of $\mathrm{Cr}$ component, each pixel value greater than threshold (mean) was classified as skin and converted to white color (with value equal to one), otherwise the pixel classified as background and converted to black color (with value equal to zero).

Threshold $=\frac{1}{w x h} \sum_{i=0}^{w-1} \sum_{j=0}^{h-1} \operatorname{Cr}(i, j)$

Where $\mathrm{w}$, and $\mathrm{h}$ are the width and height of the image, and $(i, j)$ is the pixel coordinate. The result of this step showed in Fig. 2

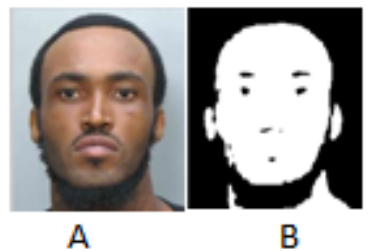

Fig. 2: creating binary image. A. color image. B. facial binary image.

The next step is to separate the image blobs in the color filtered binary image into individual regions.

Edges will be detected on binary image to divided image into separated segments achieved by applying the mask filter showed in Fig. 3 on the entire image, it scanning the image from left to right and top to down, the pixel correspond to the center of mask will be one when the result of multiplication of pixels values in the image by the corresponding values of the mask will be equal to $(1,2$, or 3 ), otherwise will be zero, Fig. 4 showed the image after edge detection.

$$
\left|\begin{array}{lll}
0 & 0 & 0 \\
0 & 1 & 1 \\
0 & 2 & 0
\end{array}\right|
$$

Fig. 3: edge detection mask

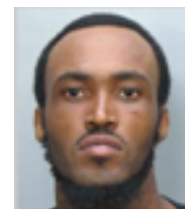

A

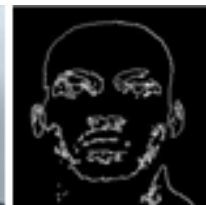

$\mathrm{B}$
Fig. 4: A. color image. B. edged image.
Gray level co-occurrence matrix (GLCM) used to remove all the regions which are not part of face. This achieved by four steps:

First: converting the origin image to grayscale image.

Second: each labeled segment in the binary image separated as separated image by leaving this labeled region white, and converting all the other regions to black. In this step the result is number of images equal to number of labeled segments.

Third: mapping each white region in the each image resulted from second step with the corresponding gray color (extracted from gray image). The result is image with gray color for specific region and black background.

Forth: applying the GLCM for each segment to extract the important features, from the extracted feature we decide which of the segment regards as not skin (not face), and then remove from the image by converting it to black color. The suggested GLCM relations for this work are.

Correlation $=\sum_{i=0}^{G-1} \sum_{j=0}^{G-1} \frac{\{i x j\} x P(i, j)-\left\{\mu_{x} * \mu\right.}{\sigma_{x} * \sigma_{y}}$

$$
\begin{aligned}
& \begin{array}{l}
\text { Inverse } \\
\sum_{i=0}^{G-1} \sum_{j=0}^{G-1} \quad \frac{1}{1+(i-j)^{2}} \quad P(i, j)
\end{array} \\
& \text { Angular Second Moment }(\mathrm{ASM})=\sum_{i=0}^{G-1} \sum_{j=0}^{G-1}\{P(i, j)\}^{2} \\
& \text { Contrast }=\sum_{n=0}^{G-1} n^{2} \quad\left\{\sum_{i=0}^{G} \sum_{j=0}^{G} P(i, j) \quad\right\} \quad,|\mathrm{i}-\mathrm{j}|=\mathrm{n}
\end{aligned}
$$

The GLCM did not remove all the non skin regions from image, actually leave some small regions in the image regards it as part of face. For that to increase the segmentation efficiency we suggested using Tamura algorithm.

One of the Tamura textures is the coarseness which is the degree of coarseness of the image. Coarseness can be calculated on colored image. The resulting images from the GLCM operations will be mapped with color image (original image) to return the corresponding color region. Coarseness can be determined by dividing the image to non-overlapping blocks. The following process will be applied to image when the block size $(2 \times 2,4 \times 4,8 \times 8$, $16 \times 16,32 \times 32$, and $64 \times 64)$.

The mean for each block will be determined by the following relation

$$
A_{k} \quad(x, y)=\frac{1}{2^{2 k}} \sum_{i=-2^{k-1}}^{2^{k-1}} \sum_{j=-2^{k-1}}^{2^{k-1}} f(x+i, y+j)
$$

Where $\mathrm{k}=1 \sim 5$ and $\mathrm{f}(\mathrm{x}+\mathrm{i}, \mathrm{y}+\mathrm{j})$ is the gray level value of $(\mathrm{x}+\mathrm{i}, \mathrm{y}+\mathrm{j})$. When $\mathrm{k}=1$, a $2 \times 2$ block is selected to calculate the average pixel value $\mathrm{A}_{1}(\mathrm{x}, \mathrm{y})$ of this block. When $\mathrm{k}=2$, a $4 \mathrm{x} 4$ block is used to calculate the average pixel value $\mathrm{A}_{2}(\mathrm{x}, \mathrm{y})$ of the block, and so on.

Later, based on each $\mathrm{k}$ value, the average gray level value difference between each pixel and its neighboring pixels

Each region segmented from this process will be labeled, (in horizontal and vertical directions) can be derived as starting from 1 which assign to first segment to the follows [10]: number of segments (regions) in the image. 


$$
\begin{aligned}
& E_{k, h}(x, y)=\left|A_{k}\left(x+2^{k-1}, y\right)-A_{k}\left(x-2^{k-1}, y\right)\right| \\
& E_{k, v}(x, y)=\left|A_{k}\left(x, y+2^{k-1}\right)-A_{k}\left(x, y-2^{k-1}\right)\right|
\end{aligned}
$$

Where (h, v) represent the horizontal and vertical directions.

Finally, after we determined $\mathrm{E}_{\mathrm{k}, \mathrm{h}}(\mathrm{x}, \mathrm{y})$, and $\mathrm{E}_{\mathrm{k}, \mathrm{v}}(\mathrm{x}, \mathrm{y})$ for $\mathrm{k}=1 \sim 5$, the maximum value $\mathrm{E}$ and the corresponding $\mathrm{k}$ are used to represent the coarseness of the pixel (x,y), denoted as $\mathrm{F}_{\text {coarse }}(\mathrm{x}, \mathrm{y})$ :

$$
\mathrm{F}_{\text {coarse }}(\mathrm{x}, \mathrm{y})=\max \left\{\mathrm{E}_{\mathrm{k}, \mathrm{i}}(\mathrm{x}, \mathrm{y}), \mathrm{i}=\mathrm{h} \text { and } \mathrm{v}, \mathrm{k}=1,2,3,4,5\right\}
$$

\section{III.}

\section{RESULTS}

The proposed algorithm tested with many images, some of them showed in Fig. 5, Fig. 6, Fig. 7, Fig. 8, Fig. 9, and Fig. 10. When we applied the proposed algorithm on each image the final image result will be either image with face (and black background) when there is face in the input image or black image when there is no face in the input image as in Fig. 8, Fig. 9, and Fig. 10. In each figure we showed the image in various stage starting from the color image (input image), $\mathrm{YCbCr}$ image, $\mathrm{Cr}$ component image, binary image, and face image ( the final result).

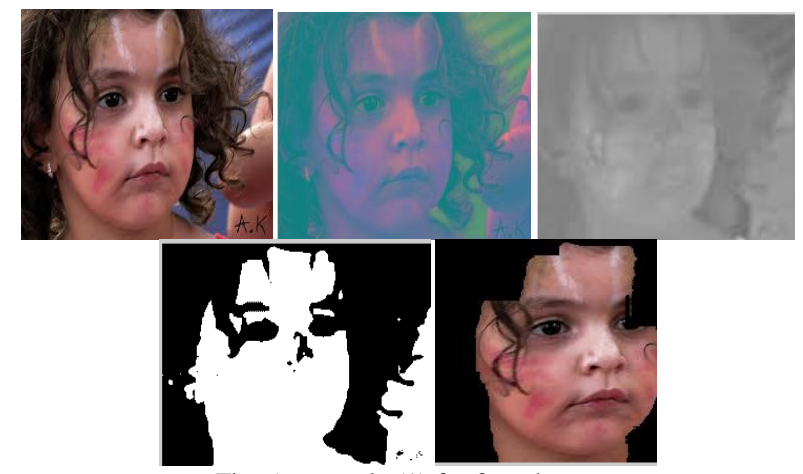

Fig. 5: example (1) for face detect
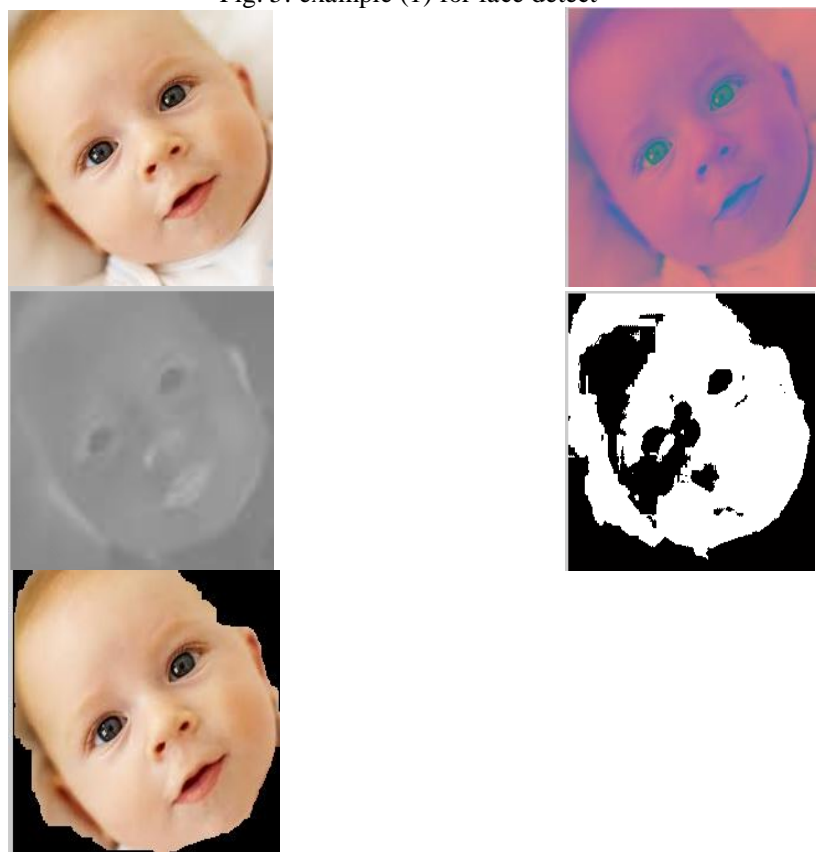

Fig. 6: example (2) for face detect
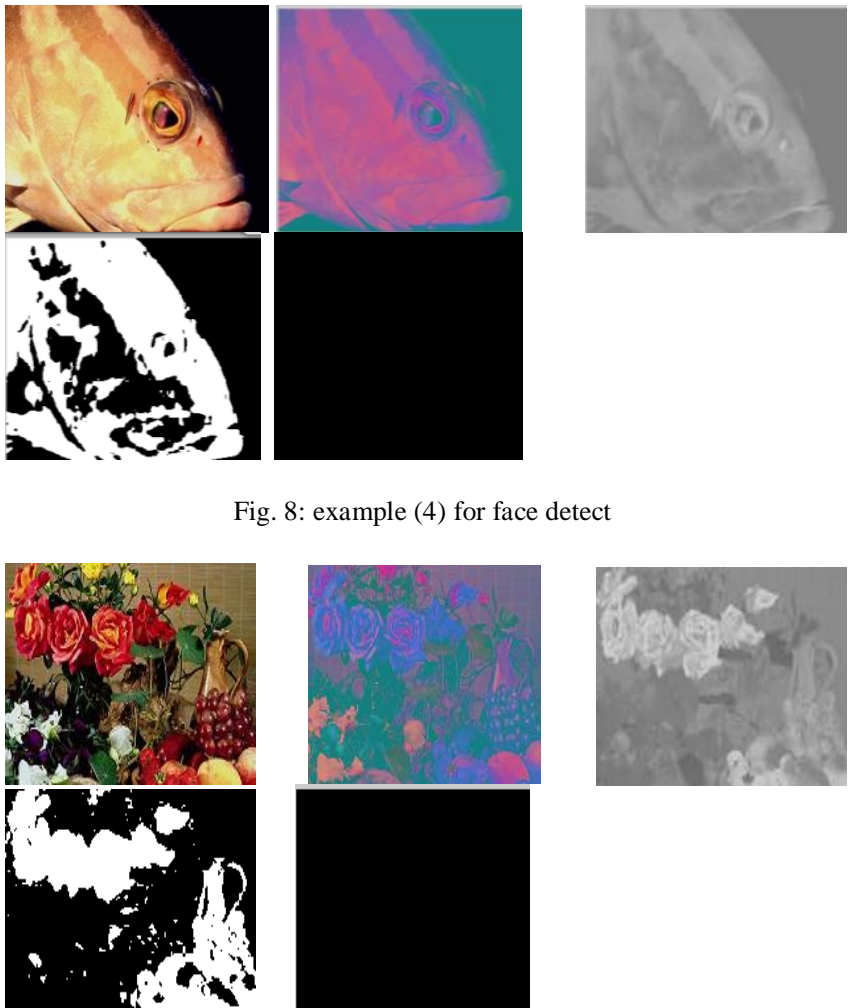

Fig. 9: example (5) for face detect
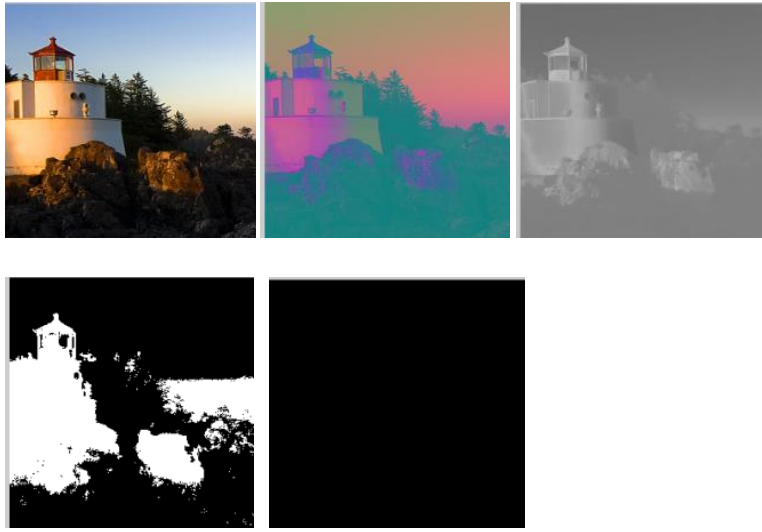

Fig. 10: example (6) for face detect

- The algorithm also checked with more than one face in the image and works well as showed in Fig. 11. 

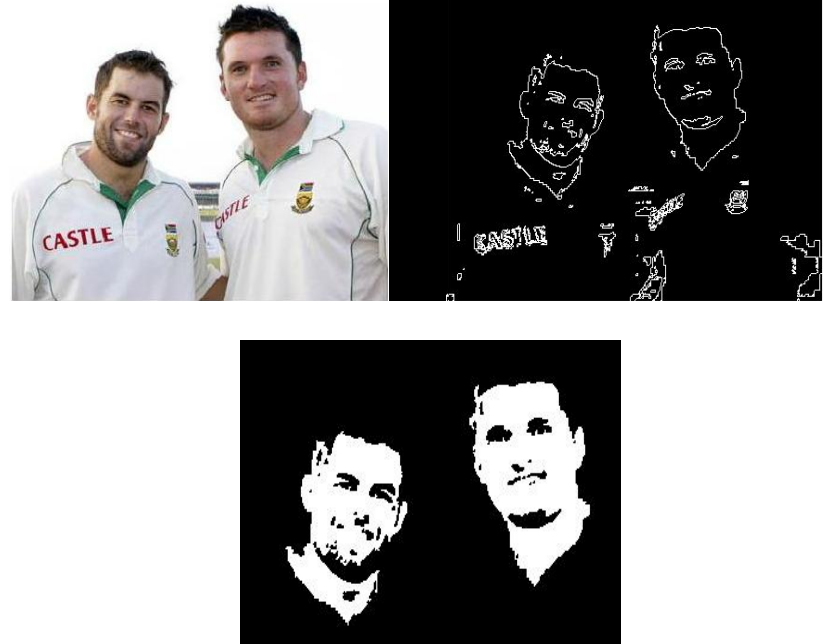

Fig. 11: image with two faces

- $\quad$ To evaluate the performance, we determined the precision and recall as follow:

Precision $=\frac{\text { No.of relevant } \text { images retrieved }}{\text { Total no.of } \text { images retrieved }} \times 100$

Precision $=\frac{100}{100} \times 100=100 \%$ for the proposed system

Recall $=\frac{\text { No.of relevant } \text { images retrieved }}{\text { Total no.of relevant images in the database }} \times 100$

Recall $=\frac{99}{100} \times 100=99 \%$ for the proposed system

- The average execution time for detection and segment the face from the image was $2.4 \mathrm{sec}$.

IV.

DISCUSSION

The algorithm tested with many images, in all images included faces it successfully detect the face and segmented it, even when there is more than one face in image.

Facial images in the experiment come mostly from digital still camera photos from life and collected stochastically from the internet.

From the other side the algorithm return black image when there is no face in image. The precision was 100\% while the recall equal $99 \%$, and this is very good result, these results better than many algorithms used to detect and segment human face. Proposed algorithm compared with other algorithms and gives promised results as shown in Table 1.

\section{CONCLUSION}

In this paper we suggested new method to detect and segment the face from an image. We suggested using the GLCM to detect the face or skin features in the image.

Also we used the Tamura to increase the segmentation efficiency, by removing the segments which is not skin and classified as skin in the GLCM.

The algorithm tested with many images and gives very good results.
We suggest expanding this work in the future to detect and segment all the human skin in the image regardless to which part of the human body belong.

In our study we applied many experimental limitations for each pixel or segment by detecting the feature rang of each face region that distinguishes it from other regions in the image. comparing proposed algorithm with other algorithms gives better results as in Table 1 .

Table 1: comparing proposed algorithm with other algorithms

\begin{tabular}{|l|l|}
\hline \multicolumn{1}{|c|}{ Algorithms } & Efficiency \\
\hline With Mixed Gaussian skin color model [6] & $92.5 \%$ \\
\hline Without Mixed Gaussian skin color model [6] & $87.5 \%$ \\
\hline $\begin{array}{l}\text { Kamarul Hawari Bin Ghazali, Jie Ma, } \\
\text { Rui Xiao [5]. }\end{array}$ & $95.45 \%$ \\
\hline Yogesh Tayal et al. 2012 & $73.68 \%$ \\
\hline Reema Ajmera, Namrata Saxena, 2013 & $84.61 \%$ \\
\hline HIS Color Space [3] & $73.8 \%$ \\
\hline YCbCr Color Space [3] & $84.61 \%$ \\
\hline RGB Color Space [3] & $21.64 \%$ \\
\hline $\begin{array}{l}\text { Hewa Majeed Zangana, Imad Fakhri } \\
\text { Shaikhli, [3] }\end{array}$ & 92.69 \\
\hline Proposed algorithm & $99 \%$ \\
\hline
\end{tabular}

\section{REFERENCES}

[1] Chaoyi Zhang, Yanning Zhang, Zenggang Lin., Automatic Face Segmentation Based on the Level Set Method, National Conference on Information Technology and Computer Science (CITCS 2012), 2012. DOI: $10.2991 /$ citcs.2012.259

[2] El Abbadi, N.K., and A.H. Miry, 2014, Automatic segmentation of skin lesions using histogram thresholding, Journal of Computer Science, 10: pp 632-639, 2014. DOI: 10.3844 /jcssp.2014.632.639.

[3] Hewa Majeed Zangana, Imad Fakhri Al-Shaikhli, ANew Algorithm for Human Face DetectionUsing Skin Color Tone, IOSR Journal of Computer Engineering, Volume 11, Issue 6, pp31-38, 2013.

[4] H. C. Vijay Lakshmi and S. Patil Kulakarni, Segmentation Algorithm for Multiple Face Detection in Color Images with Skin Tone Regions using Color Spaces and Edge Detection Techniques, International Journal of Computer Theory and Engineering, Vol. 2, No. 4, pp 1793-8201, 2010.

[5] Kamarul Hawari Bin Ghazali, Jie Ma, Rui Xiao, An Innovative Face Detection based on Skin Color Segmentation, International Journal of Computer Applications (0975 - 8887) Volume 34- No.2, 2011. DOI: $10.5120 / 4069-5244$

[6] Krishnan Nallaperumal, Ravi Subban, R. K. Selvakumar, A. Lenin Fred, Human Face Detection in Color Images Using Mixed Gaussian Color Models, International Journal of Imaging Science and Engineering (IJISE), GA, USA, ISSN: 1934-9955, VOL.2, NO.1, Jan 2008.

[7] K. Satheesh, I. Laurence Aroquiaraj, An Evaluation of Face Segmentation Algorithms, In proceeding of: EITECH 2012.

[8] Reema Ajmera, Namrata Saxena, Face Detection in Digital Images Using Color Spaces and Edge Detection Techniques, International Journal of Advanced Research in Computer Science and Software Engineering, Volume 3, Issue 6, 2013.

[9] Prof. Samir K. Bandyopadhyay, IJCSET, A Method for Face Segmentation, Facial Feature Extraction and Tracking, Vol 1, Issue 3, pp 137-139, 2011.

[10] Wan-Ting Lin, Chuen-Horng Lin, Tsung-Ho Wu, and Yung-Kuan Chan, Image Segmentation Using the K-means Algorithm for Texture Features, World Academy of Science, Engineering and Technology journal, issue 41, pp612, 2010.

[11] Yogesh Tayal, Ruchika Lamba, Subhransu Padhee, Automatic Face Detection Using Color Based Segmentation, International Journal of Scientific and Research Publications, Volume 2, Issue 6, 2012. 


\section{BIOGRAPHY}

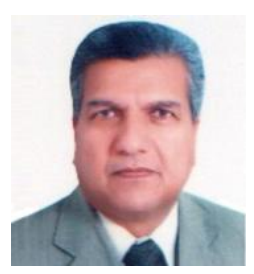

Nidhal El Abbadi, received BSc in Chemical Engineering, BSc in computer science, MSc and $\mathrm{PhD}$ in computer science, worked in industry and many universities, he is general secretary of colleges of computing and informatics society in Iraq, Member of Editorial board of Journal of Computing and Applications, reviewer for a number of international journals, has many published papers and three published books (Programming with Pascal, $\mathrm{C}++$ from beginning to OOP, Data structures in simple language), his research interests are in image processing, security, and steganography, He's Associate Professor in Computer Science in the University of KufaNajaf, IRAQ. 